\title{
SPECIFICATION AND ESTIMATION OF COBB-DOUGLAS PRODUCTION FUNCTION MODELS ${ }^{1}$
}

\author{
By A. Zellner, J. Kmenta And J. Drèze
}

\begin{abstract}
In this paper we consider the specification and estimation of the Cobb-Douglas production function model. After reviewing the "traditional" specifying assumptions for the model which are based on deterministic profit maximization, we develop a model in which profits are stochastic and in which maximization of the mathematical expectation of profits is posited. "Sampling theory" and Bayesian estimation techniques for this model are presented.
\end{abstract}

\section{INTRODUCTION}

IN THIS PAPER we take up the problem of specifying and estimating a model of a profit maximizing firm operating with a Cobb-Douglas production function. Our model differs from the traditional production model considered in the literature, in that we assume that: (a) the production process is neither instantaneous nor deterministic; and (b) entrepreneurs are aware of the stochastic nature of production in their profit maximizing endeavors.

This fundamental conceptual difference in our approach leads us to a new model with properties different from that of the traditional model. ${ }^{2}$ Also we develop both sampling theory and Bayesian estimation procedures for the new model.

The order of presentation is as follows. In Section 2 we review the traditional model, and then go on in Section 3 to formulate the new model. In Section 4, sampling theory estimation procedures are developed for the new model. In contrast with the traditional model, it is found that classical least squares provides consistent estimators of the parameters of the Cobb-Douglas production function. With a normality assumption, these are also unbiased and maximum likelihood estimators. Finally, in Section 5, a Bayesian analysis of the new model is presented.

\section{REVIEW OF THE TRADITIONAL MODEL}

According to economic theory, output, inputs, and profit of a firm are determined by the production function, the definition of profit, and the conditions of profit maximization. If the production function is of the Cobb-Douglas type with two

1 This research was supported in part by the National Science Foundation under Grants GS-151 and GS-1350, in part by the U.S. Army Mathematics Research Center, University of Wisconsin, and in part by the Social Systems Research Institute, University of Wisconsin.

${ }^{2}$ A recent paper by Mundlak and Hoch [11], which was brought to our attention after this paper had been written, considers a similar departure from the traditional model; their rationale and statistical coverage are different from ours. See footnotes 9 and 11 below. 
inputs, ${ }^{3}$ the production model of the firm would be represented as follows:

$$
\begin{array}{rlrl}
X & =A L^{\alpha_{1}} K^{\alpha_{2}} & & \text { Production function, } \\
\pi & =p X-w L-r K & & \text { Profit definition, } \\
\frac{\partial \pi}{\partial L} & =0 & & \\
\frac{\partial \pi}{\partial K} & =0 & \text { Maximizing conditions. }
\end{array}
$$

Here $\pi$ represents profits; $X, L$, and $K$ represent quantities of output and labor and capital inputs, respectively; and $p, w$, and $r$ their respective prices.

In empirical work concerned with estimation of production function parameters from cross-section data, the deterministic form of the model has been modified by the introduction of stochastic disturbances. Following Marschak and Andrews [9], the traditional representation of the production model has been: ${ }^{4}$

$$
\begin{aligned}
x_{0 i}-\alpha_{1} x_{1 i}-\alpha_{2} x_{2 i} & =\lambda_{0}+v_{0 i}, \\
x_{0 i}-x_{1 i} & =\lambda_{1}+v_{1 i}, \\
x_{0 i}-x_{2 i} & =\lambda_{2}+v_{2 i},
\end{aligned}
$$

where the subscript $i$ refers to the $i$ th firm, $x_{0 i}=\log X_{i}, x_{1 i}=\log L_{i}, x_{2 i}=\log K_{i}$, $\lambda_{0}=\log A$, and $v_{0 i}, v_{1 i}$, and $v_{2 i}$ are stochastic disturbances, assumed to have zero means and variances (and possibly nonzero covariances) which are the same for different firms. The parameters $\lambda_{1}$ and $\lambda_{2}$ are given by

$$
\lambda_{1}=\log \frac{w R_{1}}{p \alpha_{1}} \quad \text { and } \quad \lambda_{2}=\log \frac{r R_{2}}{p \alpha_{2}} \text {. }
$$

It should be noted that $\lambda_{1}$ and $\lambda_{2}$ are the same for all firms, since it is assumed that prices of output and inputs are the same for all firms. The parameters $R_{1}$ and $R_{2}$, suggested by Hoch [5], are introduced to allow for the possibility that firms in the sample may exhibit systematic errors, perhaps as a result of institutional or other constraints, with respect to satisfying the first order conditions. Of course, if $R_{1}=$ $R_{2}=1$, such systematic errors are absent.

The random disturbance terms $v_{1 i}$ and $v_{2 i}$ in the traditional model are introduced to allow for random, nonsystematic errors on the part of entrepreneurs in their attempts to adjust inputs to satisfy the necessary conditions for profit maximization. On the other hand, the interpretation of $v_{0 i}$ has not been as clearcut in the literature. Marschak and Andrews [9, p. 145 and p. 156] describe $v_{0 i}$ as reflecting

${ }^{3}$ A two-input case is used here in order to economize on exposition. The argument and the estimation methods developed in this paper can be readily extended to any number of inputs.

${ }^{4}$ The specification of the model given in the text is a special case of the imperfectly competitive model considered by Marschak and Andrews. It can be found in Klein [7], Hoch [5], Walters [14], Mundlak [10], and Kmenta [8]. 
"technical efficiency" and depending "on the technical knowledge, the will, effort and luck of a given entrepreneur." One way of making these observations regarding $v_{0 i}$ more explicit is to assume that the production functions of all firms are identical up to a neutral disembodied productivity differential; in other words, the parameters $\alpha_{1}$ and $\alpha_{2}$ are assumed common to all firms in the sample, but the parameter $A$ varies from firm to firm with $A_{i}=A_{0} e^{v_{0 i}^{+}}$, where $A_{0}$ is a common parameter, and $v_{0 i}^{+}$ is a random variable. Without loss of generality, we can assume $E v_{0 i}^{+}=0$. Then $v_{0 i}=$ $v_{0 i}^{+}+v_{0 i}^{*}$, where $v_{0 i}^{*}$ is an additional random element, with zero mean, introduced to allow for random factors affecting output, such as luck, etc. ${ }^{5}$ If this interpretation of the disturbance $v_{0 i}$ is accepted, then it should be recognized that the variance of $v_{0 i}$ will depend on the variances of $v_{0 i}^{+}$and $v_{0 i}^{*}$, and that with a single crosssection of data it will not be possible to identify these two variance components. As is well known, more than one cross-section of data is needed to estimate the components of variance. Further, if this interpretation of $v_{0 i}$ is accepted, the production function and profit function for the individual entrepreneur are stochastic even if he knows $A_{i}$. Thus, the rationale for assuming deterministic profit maximization, as is done in the traditional approach, is at variance with the above interpretation of $v_{0 i}$, an interpretation which appears to be consistent with that in the literature.

With this said about $v_{0 i}, v_{1 i}$, and $v_{2 i}$, it is clear from (2.5)-(2.7) that $x_{1 i}$ and $x_{2 i}$ are not independent of $v_{0 i}$, since each input is a function of all disturbances of the system. Consequently, classical least squares estimates of the production function parameters will be, in general, biased and inconsistent. This conclusion led to the development of a number of alternative estimation methods based on various assumptions concerning the profit maximizing conditions. In particular, the socalled "factor shares" method" ${ }^{6}$ assumes absence of profit maximizing restraints (i.e., $R_{1}=R_{2}=1$ ), and the maximum likelihood $\operatorname{method}^{7}$ requires the assumption that $v_{1 i}$ and $v_{2 i}$ are distributed independently of $v_{0 i}{ }^{8}$

\section{THE NEW MODEL}

An alternative specification, developed in the present paper, ${ }^{9}$ involves assuming that the production function of firm $i$ is stochastic, being defined by:

$$
X_{i}=A L_{i}^{\alpha_{1}} K_{i}^{\alpha_{2}} e^{u_{0 i}},
$$

${ }^{5}$ See Walters [14, p. 14], where a similar interpretation of the error term in the production function is given.

${ }^{6}$ See Klein [7], pp. 193-196.

${ }^{7}$ See Kmenta [8]. Maximum likelihood estimates are identically equivalent to those obtained by "indirect least squares," "moments" or "covariance matrix," and Hoch's generalized methods.

${ }^{8}$ If $v_{0 i}$ represents "the technical knowledge, the will, effort and luck of a given entrepreneur," as Marschak and Andrews state, the assumption that $v_{0 i}$ and $v_{1 i}$ and $v_{2 i}$ are independently distributed may be questionable.

${ }^{9}$ Basically the same statistical specification is considered by Mundlak and Hoch (section 3 of [11]), when they assume that the production function disturbance is not "transmitted" to inputs- 
where $u_{0 i}$ is a random disturbance ${ }^{10}$ representing factors such as weather, unpredictable variations in machine or labor performance, and so on. Whenever the production process is not instantaneous, the effect of the disturbance on output cannot be known until after the preselected quantities of inputs have been employed in production. Any given level of inputs will result in an uncertain quantity of output and, consequently, in an uncertain profit. The concept of "profit maximization," which is unambiguous within the deterministic framework of the model postulated by economic theory, needs a more subtle interpretation when stochastic elements are introduced: obviously no manager can maximize something which is uncertain and beyond his control.

In this paper, we assume that: (a) entrepreneurs maximize the mathematical expectation of profit; ${ }^{11}$ (b) the prices $(p, w, r)$ are either known with certainty ${ }^{12}$ or statistically independent of the production function disturbance, with expectations $\left(p_{i}^{+}, w_{i}^{+}, r_{i}^{+}\right)$for firm $i .^{13}$

The profit maximizing conditions are then

$$
\begin{aligned}
& \frac{\partial E(\pi)}{\partial L}=0, \\
& \frac{\partial E(\pi)}{\partial K}=0, \text { where }
\end{aligned}
$$

either because the firm does not maximize profits (case B1 in [11]), or because firms maximize profits while assuming that $u_{0 i}=0$ in (3.1) (case B2 in [11]). As will be seen, our rationale is quite different, but our results are consistent with those of [11]. The interesting generalization considered in section 4 of [11] would apply to our specification as well.

${ }^{10}$ The disturbances in this section are designated with the letter $u$ instead of $v$, as in Section 1 , to emphasize the difference between the two models.

${ }^{11}$ Hoch [5] noted in passing that entrepreneurs may be maximizing "anticipated" profit, but the notion was not further developed. There is no indication in Hoch's paper that "anticipated" profit should be interpreted as the "mathematical expectation" of profit; more likely, the author had in mind "next period's" profit, which is, of course, also stochastic. P. J. Dhrymes [3] considered using the nonstochastic part of the production function, but not necessarily the mathematical expectation of output. The difficulty resulting from uncertainty concerning output was noted by Yair Mundlak [10], but the specific case of maximizing the mathematical expectation of profit was not developed. In any case, Mundlak did not consider this difficulty to be serious enough to reject the traditional formulation of the production model (ibid., pp. 147-149). Mundlak and Hoch [11] recognize uncertainty, but assume that entrepreneurs behave as if production were nonstochastic with $u_{0 i}=0$.

12 This is a natural assumption for inputs-which are typically purchased before they are used in production-but not so much for output, since a stochastic output cannot be sold on a future's market.

${ }^{13}$ We are indebted to George $\mathbf{J}$. Stigler for bringing to our attention the role of this assumption. If one were assuming that output prices are related to the quantity sold (imperfect competition), the assumption that $p$ is statistically independent of $u_{0 i}$ would not be tenable, and a more subtle analysis would be needed with $E(p X) \neq p^{+} E(X)$. 


$$
E(\pi)=p^{+} E(X)-w^{+} L-r^{+} K .
$$

Now, assuming that the disturbance in the production function is normally distributed, we have

$$
E(X)=A L^{\alpha_{1}} K^{\alpha_{2}} e^{\left(\frac{1}{2}\right) \sigma_{00}},
$$

where $\sigma_{00}$ is the variance of the production function disturbance. ${ }^{14}$

In reality, the profit maximizing conditions are not likely to be exactly fulfilled because of managerial errors originating in inertia, ignorance, etc. These errors, resulting in deviations from the desirable position, can be assumed to be randomly distributed over all firms in the industry. Another source of deviations from optimality ex post is the difference between anticipated and realized prices. Let us again assume that these differences are randomly distributed over firms; more precisely, let

$$
\begin{aligned}
& \log \left(\frac{w^{+}}{p^{+}}\right)_{i}=\log \frac{w}{p}+u_{1 i}^{+} \quad \text { and } \\
& \log \left(\frac{r^{+}}{p^{+}}\right)_{i}=\log \left(\frac{r}{p}\right)+u_{2 i}^{+},
\end{aligned}
$$

where $p, w$, and $r$ are realized prices, and $u_{1}^{+}$and $u_{2}^{+}$are random differences. The latter will disappear if prices are known with certainty.

The profit maximizing conditions given by (3.1) and (3.2) should then be extended to include the two sets of disturbances. Let the disturbances resulting from managerial inertia be $u_{1 i}^{*}$ and $u_{2 i}^{*}$ and let $u_{r i}=u_{r i}^{*}+u_{r i}^{+}(r=1,2)$. Then the "new" production model can be written as

$$
\begin{aligned}
x_{0 i}-\alpha_{1} x_{1 i}-\alpha_{2} x_{2 i} & =\alpha_{0}+u_{0 i}, \\
x_{0 i}-x_{1 i} & =k_{1}^{\prime}+u_{0 i}+u_{1 i}, \\
x_{0 i}-x_{2 i} & =k_{2}^{\prime}+u_{0 i}+u_{2 i},
\end{aligned}
$$

where $\alpha_{0}=\log A, k_{1}^{\prime}=\log \left(\frac{w R_{1}}{p \alpha_{1}}\right)-\frac{1}{2} \sigma_{00}, k_{2}^{\prime}=\log \left(\frac{r R_{2}}{p \alpha_{2}}\right)-\frac{1}{2} \sigma_{00}$, and $u_{0 i}, u_{1 i}$, and $u_{2 i}$ are stochastic disturbances. The remaining symbols have the same meaning as in equations (2.5) to (2.7) of the previous section.

One important implication of the new model is that inputs do not depend on the

${ }^{14}$ It should be noted that the assumption of normality of the production function disturbance $u_{0 i}$ implies a positive relationship between $E(\pi)$ and the variance of the disturbance. When optimal input quantities are substituted in the expression for expected profit, the latter is proportional to $\exp \sigma_{00} / 2\left(1-\alpha_{1}-\alpha_{2}\right)$. The positive association between $E(\pi)$ and $\sigma_{00}$ would of course disappear if one assumed that $E\left(e^{u_{0 i}}\right)=1$, instead of assuming normality of $u_{0 i}$. 
disturbance in the production function. This is clearly seen by referring to the reduced form equations of the model, which are:

$$
\begin{aligned}
x_{0 i}= & {\left[\alpha_{0}-\alpha_{1} k_{1}^{\prime}-\alpha_{2} k_{2}^{\prime}+\left(1-\alpha_{1}-\alpha_{2}\right) u_{0 i}\right.} \\
& \left.-\alpha_{1} u_{1 i}-\alpha_{2} u_{2 i}\right] /\left(1-\alpha_{1}-\alpha_{2}\right), \\
x_{1 i}= & {\left[\alpha_{0}+\left(\alpha_{2}-1\right) k_{1}^{\prime}-\alpha_{2} k_{2}^{\prime}+\left(\alpha_{2}-1\right) u_{1 i}-\alpha_{2} u_{2 i}\right] /\left(1-\alpha_{1}-\alpha_{2}\right), } \\
x_{2 i}= & {\left[\alpha_{0}-\alpha_{1} k_{1}^{\prime}+\left(\alpha_{1}-1\right) k_{2}^{\prime}-\alpha_{1} u_{1 i}+\left(\alpha_{1}-1\right) u_{2 i}\right] /\left(1-\alpha_{1}-\alpha_{2}\right) . }
\end{aligned}
$$

A reasonable specifying assumption is that the correlations of the $u_{0 i}$ and $u_{1 i}$ and of $u_{0 i}$ and $u_{2 i}$ are both zero, since $u_{0 i}$ results largely from "acts of nature" such as weather conditions and machine performance, whereas $u_{1 i}$ and $u_{2 i}$ are due to "human errors." Under this assumption, the result that simple least squares estimates of (3.6) are inconsistent is no longer valid. Clearly, in the new model with the assumptions that $E\left(u_{0 i} u_{1 i}\right)=E\left(u_{0 i} u_{2 i}\right)=0$, simple least squares estimators are consistent; under normality, or with the stronger assumption that $u_{1 i}$ and $u_{2 i}$ are statistically independent of $u_{0 i}$, they are also unbiased. ${ }^{15}$

Another implication of the new model relates to the properties of the "factor shares" estimates. Within the context of the traditional model, the "factor shares" method leads to unbiased estimates of $\log \alpha_{1}$ and $\log \alpha_{2}$, providing that $R_{1}=R_{2}=1$. This is no longer true within the framework of our alternative model: the "factor shares" estimate of $\log \alpha_{1}$ is

$$
\log \alpha_{1}=\frac{1}{n} \sum_{i=1}^{n} \log \left(\frac{w L_{l}}{p X_{i}}\right)
$$

But from (3.5) we have, after substituting unity for $R_{1}$,

$$
\log \left(\frac{w L_{i}}{p X_{i}}\right)=\log \alpha_{1}-u_{0 i}-u_{1 i}+\left(\frac{1}{2}\right) \sigma_{00}
$$

Consequently,

$$
E\left(\log \tilde{\alpha}_{1}\right)=\log \alpha_{1}+\left(\frac{1}{2}\right) \sigma_{00} \neq \log \alpha_{1} .
$$

Thus, the "factor shares" estimator of $\log \alpha_{1}$ has an upward bias, and

$$
\operatorname{antilog} E\left(\log \tilde{\alpha}_{1}\right)=\alpha_{1} e^{\sigma_{00 / 2}} \text {. }
$$

Similarly,

$$
\operatorname{antilog} E\left(\log \tilde{\alpha}_{2}\right)=\alpha_{2} e^{\sigma_{00 / 2}} .
$$

Thus, the ratio of the antilogs of the two expectations is equal to true ratio $\alpha_{1} / \alpha_{2}$, 
but the sum of the antilogs, $\left(\alpha_{1}+\alpha_{2}\right) e^{\left(\frac{1}{2}\right)} \sigma_{00}$ is not equal to the returns to scale parameter. The bias in estimating returns to scale is a natural consequence of the stochastic nature of the production function: the numerators of the factor share estimates are uncorrelated with $u_{0}$ but the denominators are-which has an effect similar (but opposed in sign) to that of a measurement error or "transitory component" in a regressand. ${ }^{16}$

A final point worth noting concerns the maximum likelihood estimates derived for the traditional model. These estimates are identified by the assumption that $\left(x_{0 i}-x_{1 i}\right)$ and $\left(x_{0 i}-x_{2 i}\right)$ are independent of the disturbance in the production function. But in our model it follows, from equations (3.7) and (3.8), that $E\left(x_{0 i}-x_{1 i}\right) u_{0 i}=E\left(x_{1 i}-x_{2 i}\right) u_{0 i}=\sigma_{00}(\neq 0)$, even if $u_{1 i}$ and $u_{2 i}$ are independent of $u_{0 i}$.

\section{MAXIMUM LIKELIHOOD ESTIMATION OF THE NEW MODEL}

In the previous section we argued that the disturbances in the profit maximizing conditions, $u_{1 i}$ and $u_{2 i}$, can be expected to be independent of the disturbance in the production function, $u_{0 i}$. If this is true, and if firms' disturbances are normally and independently distributed with zero means and constant covariance matrix, we can derive the maximum likelihood estimates of the production function parameters as follows. ${ }^{17}$

The system, described by equations (3.6) to (3.8), can be rewritten as

$$
\begin{aligned}
& x_{0 i}-\alpha_{1} x_{1 i}-\alpha_{2} x_{2 i}-\alpha_{0}=u_{0 i}, \\
& \left(\alpha_{1}-1\right) x_{1 i}+\alpha_{2} x_{2 i}-k_{1}=u_{1 i}, \\
& \alpha_{1} x_{1 i}+\left(\alpha_{2}-1\right) x_{2 i}-k_{2}=u_{2 i},
\end{aligned}
$$

where

$$
k_{1}=\log \frac{w R_{1}}{A \alpha_{1} p}-\frac{\sigma_{00}}{2}+\alpha_{0}, \quad k_{2}=\log \frac{r R_{1}}{A \alpha_{2} p}-\frac{\sigma_{00}}{2}+\alpha_{0},
$$

and $\alpha_{0}=\log A$.

The variance-covariance matrix of the disturbances is

$$
\Sigma=\left[\begin{array}{c:cc}
\sigma_{00} & 0 & 0 \\
\hdashline 0 & \Sigma_{*} \\
0 & \ldots
\end{array}\right],
$$

where

$$
\Sigma_{*}=\left[\begin{array}{ll}
\sigma_{11} & \sigma_{12} \\
\sigma_{12} & \sigma_{22}
\end{array}\right] .
$$

${ }^{16}$ The analogy would be perfect if one were trying to estimate returns to scale by regressing cost on output.

${ }_{17}$ Subject to the condition that $\alpha_{1}+\alpha_{2} \neq 1$, and $\alpha_{1}, \alpha_{2}>0$. As pointed out by $T$. Takayama and G. G. Judge, this prior information is not incorporated in "conventional" maximum likelihood estimates. 
Further, let $u_{r}(r=0,1,2)$ be a $(n \times 1)$ vector of the random disturbances. Then the logarithm of the likelihood function is given by

$$
\begin{aligned}
L= & -\frac{3 n}{2} \log (2 \pi)+n \log |J|-\frac{n}{2} \log |\Sigma| \\
& -\frac{1}{2} \frac{\left(u_{0}^{\prime} u_{0}\right)}{\sigma_{00}}-\frac{1}{2}\left(u_{1}^{\prime} u_{2}^{\prime}\right)\left(\Sigma_{*}^{-1} \otimes I_{n}\right)\left(\begin{array}{l}
u_{1} \\
u_{2}
\end{array}\right),
\end{aligned}
$$

where $|J|=\left|1-\alpha_{1}-\alpha_{2}\right|$ is the Jacobian for the transformation from the $u$ 's to the $x$ 's given in (4.1)-(4.3). Differentiating $L$ with respect to the unknown parameters and putting the first derivatives equal to zero leads to simple least squares estimates of $\alpha_{1}$ and $\alpha_{2}$. The estimates of the nonzero elements of $\Sigma$ are

$$
\begin{array}{ll}
\hat{\sigma}_{r r}=\frac{1}{n} \hat{u}_{r}^{\prime} \hat{u}_{r}, & (r=0,1,2) \\
\hat{\sigma}_{12}=\frac{1}{n} \hat{u}_{1}^{\prime} \hat{u}_{2}, &
\end{array}
$$

where $\hat{u}_{r}(r=0,1,2)$ is a vector of residuals.

Since each of the $x$ 's can be expressed in terms of the disturbances, as per (3.9)(3.11), $\hat{\alpha}_{1}$ and $\hat{\alpha}_{2}$-the simple least squares estimates of $\alpha_{1}$ and $\alpha_{2}$-can be similarly expressed. This gives

$$
\begin{aligned}
\hat{\alpha}_{r}=\alpha_{r} & +\left[\left(\alpha_{r}+r-2\right)\left(u_{2}^{\prime} u_{2}\right)-\left(\alpha_{r}-r+1\right)\left(u_{1}^{\prime} u_{2}\right)\right]\left(\frac{u_{0}^{\prime} u_{1}}{D}\right) \\
- & {\left[\left(\alpha_{r}+r-2\right)\left(u_{1}^{\prime} u_{2}\right)-\left(\alpha_{r}-r+1\right)\left(u_{1}^{\prime} u_{1}\right)\right]\left(\frac{u_{0}^{\prime} u_{2}}{D}\right), }
\end{aligned}
$$

where $D=\left(u_{1}^{\prime} u_{1}\right)\left(u_{2}^{\prime} u_{2}\right)-\left(u_{1}^{\prime} u_{2}\right)^{2}$, and $r=1,2$. From this it immediately follows that $\hat{\alpha}_{1}$ and $\hat{\alpha}_{2}$ are unbiased and consistent. Further, it can easily be shown that the estimates of the variances of $\hat{\alpha}_{1}$ and $\hat{\alpha}_{2}$ calculated in the conventional way are asymptotically equal to

$$
n^{-1} \lim \underset{n \rightarrow \infty}{E}\left[\sqrt{n}\left(\hat{\alpha}_{r}-\alpha_{r}\right)\right]^{2}, \quad(r=1,2) .
$$

From the classical sampling theory point of view, the new model thus vindicates the single-equation approach to estimation of the Cobb-Douglas function from cross-section data. The profit maximizing conditions are interesting as a means of testing for the efficient average allocation of resources. As far as estimation of the production function parameters is concerned, the simultaneous nature of the model is relevant only because it makes $x_{1}$ and $x_{2}$ stochastic. As a result, the finite sample properties of the least squares estimators, except for their property of unbiasedness, remain to be established. 


\section{BAYESIAN ANALYSIS OF THE NEW MODEL}

In the Bayesian analysis of the new model, we employ the following "minimal information" or "noninformative" prior distribution for the parameters of the model, $\alpha=\left(\alpha_{1}, \alpha_{2}, \alpha_{0}\right), k=\left(k_{1}, k_{2}\right), \sigma_{00}$ and $\Sigma_{*}$ :

$$
p\left(\alpha, k, \sigma_{00}, \Sigma_{*}\right) \propto p_{1}(\alpha, k) p_{2}\left(\sigma_{00}\right) p_{3}\left(\Sigma_{*}\right),
$$

where

$$
\begin{aligned}
& p_{1}(\alpha, k) \propto\left|1-\alpha_{1}-\alpha_{2}\right|^{-1}, \\
& p_{2}\left(\sigma_{00}\right) \propto 1 / \sigma_{00}, \text { and } p_{3}\left(\Sigma_{*}\right) \propto\left|\Sigma_{*}\right|^{-\frac{3}{2}} .
\end{aligned}
$$

In (5.1) we follow Jeffreys [6] and Box and Tiao [1] in taking location and scale parameters to be independently distributed. Further, (5.2b) expresses ignorance about $\sigma_{00}$ and $\Sigma$ * in accord with Jeffreys' invariance theory [6, p. $179 \mathrm{ff}$.] and in a way consistent with the approach employed by Savage [13]. As regards (5.2a), we take $\alpha_{0}, k_{1}$, and $k_{2}$ to be uniformly and independently distributed. Further, the prior density of $\alpha_{1}$ and $\alpha_{2}$ is taken proportional to $\left|1-\alpha_{1}-\alpha_{2}\right|^{-1}$, a result provided by application of Jeffreys' invariance theory.

Now we use Bayes' Theorem to combine the prior distribution in (5.1) with the likelihood function for the model to obtain the following joint posterior distribution of the parameters:

$$
p\left(\alpha, k, \sigma_{00}, \Sigma_{*} \mid S\right) \propto \frac{\left|1-\alpha_{1}-\alpha_{2}\right|^{n-1}}{\sigma_{00}^{(n+2) / 2}\left|\Sigma_{*}\right|^{(n+3) / 2}} \exp \left\{-\frac{1}{2} u_{0}^{\prime} u_{0} / \sigma_{00}-\frac{1}{2} \operatorname{tr} A \Sigma_{*}^{-1}\right\},
$$

where $S$ denotes the sample information; $u_{0}, u_{1}$, and $u_{2}$, each $n \times 1$ column vectors, are defined by the equations of the new model in (4.1)-(4.3); and

$$
A=\left(\begin{array}{cc}
u_{1}^{\prime} u_{1} & u_{1}^{\prime} u_{2} \\
u_{2}^{\prime} u_{1} & u_{2}^{\prime} u_{2}
\end{array}\right) .
$$

On integrating over $\sigma_{00}$ and the elements of $\Sigma_{*}$, we obtain the following joint posterior distribution for the parameters $\alpha$ and $k$ :

$$
p(\alpha, k \mid S) \propto \frac{\left|1-\alpha_{1}-\alpha_{2}\right|^{n-1}}{\left(u_{0}^{\prime} u_{0}\right)^{n / 2}|A|^{n / 2}} .
$$

Noting that $|A|$ is quadratic in $k_{1}$ and $k_{2}$, these parameters can be easily integrated out to yield:

$$
p\left(\alpha_{1}, \alpha_{2}, \alpha_{0} \mid S\right) \propto \frac{\left|1-\alpha_{1}-\alpha_{2}\right|^{n-1}}{\left(u_{0}^{\prime} u_{0}\right)^{n / 2}\left|A^{*}\right|^{(n-1) / 2}},
$$

where

$$
A^{*}=\left[\begin{array}{ll}
\left(u_{1}-\bar{u}_{1} \iota\right)^{\prime}\left(u_{1}-\bar{u}_{1} \iota\right) & \left(u_{1}-\bar{u}_{1} \iota\right)^{\prime}\left(u_{2}-\bar{u}_{2} \iota\right) \\
\left(u_{2}-\bar{u}_{2} \iota\right)^{\prime}\left(u_{1}-\bar{u}_{1} \iota\right) & \left(u_{2}-\bar{u}_{2} \iota\right)^{\prime}\left(u_{2}-\bar{u}_{2} \iota\right)
\end{array}\right]
$$


with $\bar{u}_{r}=n^{-1} \sum_{i=1}^{n} u_{r i}$ for $r=1,2$, and $\iota$ is a $n \times 1$ column vector of ones, i.e., $\iota^{\prime}=$ $(1,1, \ldots, 1)$. We note that

$$
A^{*}=\left(\begin{array}{c:c}
\alpha_{1}-1 & \alpha_{2} \\
\hdashline \alpha_{1} & \alpha_{2}-1
\end{array}\right)\left(\begin{array}{c}
x_{1}^{\prime}-\bar{x}_{1} \iota^{\prime} \\
\hdashline x_{2}^{\prime}-\bar{x}_{2} \iota^{\prime}
\end{array}\right)\left(x_{1}-\bar{x}_{1} \iota x_{2}-\bar{x}_{2} \iota\right)\left(\begin{array}{c:c}
\alpha_{1}-1 & \alpha_{1} \\
\hdashline \alpha_{2} & \alpha_{2}-1
\end{array}\right),
$$

and thus $\left|A^{*}\right| \propto\left(1-\alpha_{1}-\alpha_{2}\right)^{2}$. With this fact noted, (5.5) becomes:

$$
\begin{aligned}
p\left(\alpha_{1}, \alpha_{2}, \alpha_{0} \mid S\right) & \propto\left(u_{0}^{\prime} u_{0}\right)^{-n / 2} \\
& \propto\left[v S^{2}+(\alpha-\hat{\alpha})^{\prime} X^{\prime} X(\alpha-\hat{\alpha})\right]^{-(v+3) / 2}
\end{aligned}
$$

where $X=\left(x_{1} x_{2} \iota\right), v=n-3, \hat{\alpha}=\left(X^{\prime} X\right)^{-1} X^{\prime} x_{0}$, and $s^{2}=v^{-1}\left(x_{0}-X \hat{\alpha}\right)^{\prime}\left(x_{0}-X \hat{\alpha}\right)$. From (5.6) it is apparent that the posterior distribution of the production functions' parameters, $\alpha_{0}, \alpha_{1}$, and $\alpha_{2}$, is in the form of a trivariate $t$ distribution. ${ }^{18}$ From this fact it follows that:

(i) $\frac{\alpha_{i}-\hat{\alpha}_{i}}{s \sqrt{h^{i i}}}$ has a univariate $t$ distribution with $v=n-3$ degrees of freedom, where $\hat{\alpha}_{i}(i=0,1,2)$ is the least squares quantity, and $h^{i i}$ is the $(i, i)$ th element of $\left(X^{\prime} X\right)^{-1}$;

and

(ii) $\alpha_{1}$ and $\alpha_{2}$ have a posterior distribution in the bivariate $-t$ form $;^{19}$

(iii) the returns to scale parameter, say $\omega$, defined as $\omega=\alpha_{1}+\alpha_{2}$, is distributed in the univariate $t$ form; that is, the quantity

$$
t=\frac{\omega-\left(\hat{\alpha}_{1}+\hat{\alpha}_{2}\right)}{s_{\omega}}
$$

has a univariate $t$ distribution with $n-3$ degrees of freedom, where

$$
s_{\omega}=s \sqrt{\frac{m_{11}+m_{22}-2 m_{12}}{m_{11}+m_{22}-m_{12}^{2}}}
$$

and $m_{r r^{\prime}}=\left(x_{r}-\bar{x}_{r} \iota\right)^{\prime}\left(x_{r^{\prime}}-\bar{x}_{r^{\prime}} \iota\right)\left(r, r^{\prime}=1,2\right)$ are sample moments about sample means.

The generalization of these results to the case of more inputs is quite direct and thus will not be developed here. In this case, the parameters of the production function are again distributed a posteriori in the multivariate $t$ form. Further, the returns to scale parameter is distributed in the univariate $t$ form. Thus, all the required distributional results for making exact finite sample inferences are available.

${ }^{18}$ For properties of the multivariate $t$ distribution, see Cornish [2], Dunnet and Sobel [4], and Raiffa and Schlaifer [12].

${ }^{19}$ If the prior information, $\alpha_{1}>0, \alpha_{2}>0$ and $0<\alpha_{1}+\alpha_{2}<1$, were introduced as a uniform prior distribution, the posterior distribution for $\alpha_{1}$ and $\alpha_{2}$ would be truncated. Numerical techniques for analyzing such a distribution are readily available. 


\section{CONCLUSION}

Above, we have presented two alternative specifications of the Cobb-Douglas production function model. The first, which we refer to as the "traditional model," assumes that firms operate on a nonstochastic production function and maximize profits. The model is then made stochastic by introduction of random disturbance terms by the econometrician, which yields the traditional or Marschak-Andrews' model.

The second model which we have considered involves assuming that the production functions of firms are identical insofar as form and parameters are concerned and, in addition, are stochastic. In this model, the profit function is random, and we have assumed that firms maximize the mathematical expectation of profits, an assumption that leads to a new model for which estimating techniques have been presented above. In our opinion, it seems desirable in many economic contexts to incorporate the assumption that entrepreneurs are aware of the stochastic nature of the production process. Further, we have noted that others, mentioned above, and more recently Mundlak and Hoch [11], appear to be aware of some of the statistical properties of this model, but have failed to provide an economic rationale for it in terms of maximization of the mathematical expectation of returns. While we consider this rationalization of our model an improvement relative to deterministic profit maximization, we are fully aware of the fact that one-period maximization of expected returns is just a step in the direction of a proper treatment of stochastic elements in a firms' sequential decision-making process under uncertainty. For instance, attention should be paid to the fact that the variability of output given inputs is sometimes controllable-at some cost-so that the variance of the production function disturbance may reflect some underlying optimization process. ${ }^{20}$ In addition, even within the context of our one-period model, further generalization is possible to take account of interfirm differences. For example, as Mundlak and Hoch point out, specific nonrandom firm effects, as well as random disturbance terms, can be introduced to take account of possible neutral disembodied productivity differentials. ${ }^{21}$ Further, it would be possible to consider all or some parameters of the production function to be random, thus allowing for interfirm differences in the exponents of the Cobb-Douglas function, as well as in the multiplicative parameter. In such a specification of the model, the econometrician can estimate the means of random coefficients.

Finally, we note that in many production function analyses annual data are employed, when actual production decisions are probably made in terms of time

${ }^{20}$ This remark is not meant to apply to such sources of randomness as weather conditions; examples of situations to which it seems relevant would include control of machine performance, the choice of equipment by telephone or electricity companies, the management of processes involving waiting lines, etc.

${ }^{21}$ See [11], Section 4. 
intervals much shorter than a year. There is thus, on many occasions, an important temporal aggregation problem which should not be glossed over. In our opinion, an appropriate approach to this problem would involve analyzing the intrayear sequential decision-making process to ascertain what implications it has for the annual data. Current specifying assumptions for production models may very well be found to be seriously deficient in terms of such an analysis.

University of Chicago,

Michigan State University,

and

Université Catholique de Louvain

\section{REFERENCES}

[1] Box, G. E. P., AND G. C. TIAO: "A Further Look at Robustness via Bayes' Theorem," Biometrika, 49 (1962), 419-432.

[2] Cornish, E. A.: "The Multivariate t-Distribution Associated with a Set of Normal Sample Deviates," Australian Journal of Physics, 7 (1954), 531-542.

[3] Dhrymes, P. J.: "On Devising Unbiased Estimators for the Parameters of the Cobb-Douglas Production Function,” Econometrica, 30 (April, 1962), 297-304.

[4] Dunnett, C. W., AND M. Sobel: "A Bivariate Generalization of Student's t-Distribution with Tables for Certain Special Cases," Biometrika, 41 (1954), 153-169.

[5] Hoch, Irving: "Simultaneous Equation Bias in the Context of the Cobb-Douglas Production Function," Econometrica, 26 (October, 1958), 566-578.

[6] Jefrreys, H.: Theory of Probability (3rd edition), Oxford: Clarendon Press, 1961.

[7] Klein, L. R.: A Textbook of Econometrics, New York: Row, Peterson, and Co., 1953.

[8] Kmenta, J.: "Some Properties of Alternative Estimates of the Cobb-Douglas Production Function," Econometrica, 32 (January-April, 1964), 183-188.

[9] Marschak, J., AND W. J. Andrews: "Random Simultaneous Equations and the Theory of Production," Econometrica, 12 (July-October, 1944), 143-205.

[10] MundlaK, YaIR: "Estimation of Production and Behavioral Functions from a Combination of Cross-Section and Time-Series Data," in Christ, C. F., et al., Measurement in Economics, Stanford: Stanford University Press, 1963.

[11] MundlaK, Y., AND I. Hoch: "Consequence of Alternative Specifications in Estimation of Cobb-Douglas Production Functions," Econometrica, 33 (October, 1965), 814-828.

[12] Raiffa, H., ANd R. Schlaifer: Applied Statistical Decision Theory, Cambridge, Massachusetts: Harvard University Press, 1961.

[13] Savage, L. J.: The Subjective Basis of Statistical Practice (manuscript), Ann Arbor: University of Michigan, 1961.

[14] Walters, A. A.: "Production and Cost Functions: An Econometric Survey," Econometrica, 31 (January-April, 1963), 1-66. 